\title{
Predicting Behavior of Solitary Fibrous Tumor: Are We Getting Closer to More Accurate Risk Assessment?
}

\author{
Leona A. Doyle, MD and Christopher D. M. Fletcher, MD, FRCPath \\ Department of Pathology, Brigham and Women's Hospital, Harvard Medical School, Boston, MA
}

Predicting clinical behavior of solitary fibrous tumor (SFT) has long been a notoriously difficult task for both oncologist and surgical pathologist. It is well established that morphologically benign tumors may occasionally metastasize and conversely that many tumors, $\sim 45 \%$, classified as malignant based on histologic findings have an indolent clinical course. ${ }^{1,2}$ In this regard, SFT represents a biologic continuum with regard to both histologic appearances and clinical behavior. Although many studies have examined clinical and pathological parameters that may predict risk of relapse in SFT, the majority of these studies have examined small case numbers with limited follow-up.

In this issue of Annals of Surgical Oncology, two separate groups have attempted to define clinical and pathologic factors that may predict local recurrence and distant metastasis, by retrospective analysis of 83 and 81 patients with SFT, at a wide variety of anatomic sites, respectively. ${ }^{3,4}$ In both studies, the presence or absence of certain histologic features was gleaned from review of pathology reports. Wilky and colleagues found that anatomic site, specifically extrathoracic location, and the presence of histologic features said to be diagnostic of malignancy as described by England in 1989 (hypercellularity, $>4$ mitoses per 10 high-power fields [HPF], pleomorphism), as well as "atypical" histologic features that were considered "borderline" by Wilky et al. (0-4 mitoses per HPF, foci of hypercellularity, and atypia) were significantly associated with either local recurrence or distant metastasis. ${ }^{1,3}$ However, the relative impact of each of these histologic features was not evaluated by

(C) Society of Surgical Oncology 2013

First Received: 5 August 2013;

Published Online: 19 September 2013

C. D. M. Fletcher, MD, FRCPath

e-mail: cfletcher@partners.org multivariate analysis. They reported overall rates of 12 and $5 \%$ for local recurrence and metastasis, respectively, with a median follow-up time of 37 months. As might be expected (because of inevitably narrow or positive excision margins), the highest rate of local recurrence occurred in SFT of the CNS, which (in this study) represented $29 \%$ of the study population, but the association between extrathoracic location and increased recurrence rate persisted when CNS tumors were excluded. Although, in this study extrathoracic SFT was more likely to have atypical or malignant histologic features, the difference in recurrence rate (local or distant) seen in extrathoracic locations persisted after correction for size, histologic features, and margin status. Tumor size was not significantly associated with local recurrence or metastasis, but size $>10 \mathrm{~cm}$ was associated with malignant histology.

Van Houdt et al. ${ }^{4}$ found that tumor size $>10 \mathrm{~cm}$ and mitoses $>4$ per 10 high-power fields predicted metastasis in a cohort of 81 patients. The rates of local recurrence $(29 \%)$ and metastasis (34\%), with a median follow-up of 34 months, were substantially higher than those in the cohort studied by Wilky et al., perhaps reflecting referral bias to the tertiary centers involved in the Dutch study, as the follow-up periods were similar in duration in both studies. Local recurrence was significantly associated with positive resection margins at the time of initial surgery, reflecting the unusually high proportion of head and neck tumors in this series, accounting for $27 \%$ of cases. In contrast to Wilky and colleagues, these authors did not find a significant correlation between anatomic location and the development of recurrent disease.

With regard to anatomic site, most previous studies have not shown a significant correlation between anatomic site and the development of metastasis, although studies have been limited by small sample size and short follow-up. Approximately $10 \%$ of extrathoracic SFTs showed local recurrence or metastasis in a study by Vallat-Decouvelaere 
et al., a rate similar to that reported for thoracic SFT. ${ }^{2}$ However, one of the largest studies to examine outcome in SFT found that extrathoracic lesions had a small but significantly increased risk of local recurrence, but not of metastasis. ${ }^{5}$ Similarly, tumor size has been variably associated with increased risk of local recurrence or distant metastasis; a persistent confounding factor is that large tumor size is usually associated with malignant histology. ${ }^{2,6}$

The most consistent predictor of poor outcome in SFT has traditionally been thought to be the presence of histologic features suggestive of malignancy, such as hypercellularity, pleomorphism, mitotic count $\geq 4$ per 10 HPF, and necrosis. ${ }^{3,7}$ Notably, however, Van Houdt et al., as well as other groups, have not found a significant association between the presence of necrosis and metastasis, and, in a large study, cellularity and pleomorphism were not reliable predictors of malignant behavior. ${ }^{4-6}$ Furthermore, the subjectivity of assessing cellularity and pleomorphism makes reproducibility difficult, and thus these parameters have more limited utility than objective features such as site, size, and mitotic activity.

Difficulty with diagnostic uniformity, as well as in determining reproducible criteria for malignancy, has also constrained accurate prognostication in SFT. It is now well established that most tumors formerly classified as hemangiopericytoma (at all sites except the sinonasal tract) in fact represent SFT. ${ }^{8}$ However, for quite some time, diagnostic criteria have been imprecise (or at least hard to define), and some people feared that the diagnosis of SFT was becoming a wastebasket. The recent discovery of the seemingly specific NAB2-STAT6 gene fusion in the vast majority of SFTs, due to recurrent inversion at the 12q13 locus, will allow for more accurate and consistent classification of this tumor type. ${ }^{9,10}$ In fact, the resulting overexpression of STAT6 protein has very recently been shown to be a highly sensitive and specific immunohistochemical marker for these tumors, and this should lead to more accurate diagnosis and therefore make prognostication more reliable going forward. ${ }^{11,12}$

The conclusions drawn by Wilky and Van Houdt are similar to those reported quite recently by Demicco et al., who found that tumor size $>15 \mathrm{~cm}$, mitotic activity $\geq 4$ per $10 \mathrm{HPF}$, and patient age $\geq 55$ years predict the development of metastasis, with size and mitotic rate being the most powerful predictors after multivariate analysis. ${ }^{6}$ It therefore seems that size and mitotic activity have emerged as the most consistently reliable and objective parameters for predicting malignant behavior in SFT, confirming results from prior studies. ${ }^{1,2,5,6}$ Using these parameters, Demicco et al. ${ }^{6}$ have proposed a risk-assessment model, similar to that used in the evaluation of gastrointestinal stromal tumors, to stratify patients into three groups with low, moderate, or high risk of developing metastasis, based on patent age, tumor size, and mitotic count. In the low-risk group, no patient developed metastasis, whereas the 5- and 10 -year metastasis-free survival rates for the moderate- and high-risk groups were 77 and $64 \%$, and 15 and $0 \%$, respectively. This risk assessment model may provide a useful objective tool to more accurately predict behavior in SFT; follow-up studies to validate this method will clearly be informative, but data from the studies of Wilky and Van Houdt reported herein support the use of such a model.

\section{REFERENCES}

1. England DM, Hochholzer L, McCarthy MJ. Localized benign and malignant fibrous tumors of the pleura. A clinicopathologic review of 223 cases. Am J Surg Pathol. 1989;13:640-658.

2. Vallat-Decouvelaere AV, Dry SM, Fletcher CD. Atypical and malignant solitary fibrous tumors in extrathoracic locations: Evidence of their comparability to intra-thoracic tumors. Am J Surg Pathol. 1998;22:1501-1511.

3. Wilky BA, Montgomery EA, Guzzetta AA, Ahuja N, Meyer CF. Extrathoracic location and "borderline" histology are associated with recurrence of solitary fibrous tumors after surgical resection. Ann Surg Oncol. doi:10.1245/s10434-013-3241-x.

4. Van Houdt WJ, Westerveld CJM, Vrijenhoek JEP, van Gorp J, Verhoef C, Van Coevorden F, Van Dalen T. Prognosis of solitary fibrous tumors: A multicenter study. Ann Surg Oncol. doi:10. 1245/s10434-013-3242-9.

5. Gold JS, Antonescu CR, Hajdu C, Ferrone CR, Hussain M, Lewis $\mathrm{JJ}$, et al. Clinicopathologic correlates of solitary fibrous tumors. Cancer. 2002;94:1057-1068.

6. Demicco EG, Park MS, Araujo DM, Fox PS, Bassett RL, Pollock RE, et al. Solitary fibrous tumor: A clinicopathological study of 110 cases and proposed risk assessment model. Mod Pathol. 2012;25:1298-1306.

7. Cranshaw IM, Gikas PD, Fisher C, Thway K, Thomas JM, Hayes AJ. Clinical outcomes of extra-thoracic solitary fibrous tumors. Eur J Surg Oncol. 2009;35:994-998.

8. Fletcher CDM, Bridge JA, Hogendoorn PCW, Mertens F, eds. WHO Classification of Tumours of Soft Tissue and Bone. IARC: Lyon; 2013.

9. Robinson DR, Wu YM, Kalyana-Sundaram S, Cao X, Lonigro RJ, Sung YS, et al. Identification of recurrent NAB2-STAT6 gene fusions in solitary fibrous tumor by integrative sequencing. Nat Genet. 2013;45:180-185.

10. Chmielecki J, Crago AM, Rosenberg M, O'Connor R, Walker $\mathrm{SR}$, Ambrogio L, et al. Whole-exome sequencing identifies a recurrent NAB2-STAT6 fusion in solitary fibrous tumors. Nat Genet. 2013;45:131-132.

11. Schweizer L, Koelsche C, Sahm F, Piro RM, Capper D, Reuss $\mathrm{DE}$, et al. Meningeal hemangiopericytoma and solitary fibrous tumors carry the NAB2-STAT6 fusion and can be diagnosed by nuclear expression of STAT6 protein. Acta Neuropathol. 2013;125:651-658.

12. Doyle LA, Vivero M, Fletcher CDM, Mertens F, Hornick JL. Nuclear expression of STAT6 distinguishes solitary fibrous tumor from histologic mimics. Mod Pathol. 2013. In press. 